\title{
Moist Recirculation and Water Vapor Transport on Dry Isentropes*
}

\author{
FRÉDÉRIC LALIBERTÉ \\ Department of Physics, University of Toronto, Toronto, Ontario, Canada \\ TIFFANY SHAW \\ Columbia University, New York, New York \\ OLIVIER PAULUiS \\ Courant Institute of Mathematical Sciences, New York, New York
}

(Manuscript received 11 May 2011, in final form 16 September 2011)

\begin{abstract}
An analysis of the overturning circulation in dry isentropic coordinates using reanalysis data is presented. The meridional mass fluxes on surfaces of constant dry potential temperature but distinct equivalent potential temperature are separated into southward and northward contributions. The separation identifies thermodynamically distinct mass fluxes moving in opposite directions. The eddy meridional water vapor transport is shown to be associated with large poleward and equatorward mass fluxes occurring at the same value of dry potential temperature but different equivalent potential temperature. These mass fluxes, referred to here as the moist recirculation, are associated with an export of water vapor from the subtropics connecting the Hadley cell to the midlatitude storm tracks.

The poleward branch of the moist recirculation occurs at mean equivalent potential temperatures comparable to upper tropospheric dry potential temperature values, indicating that typical poleward-moving air parcels can ascend to the tropopause. The analysis suggests that these air parcels ascend on the equatorward side of storm tracks by following moist isentropes reminiscent of upright deep convection, while on the poleward side their moist isentropes are indicative of large-scale slantwise convection. In the equatorward branch, the analysis describes typical air parcels that follow their dry isentropes until they get injected into the boundary layer where they are subsequently moistened.

The moist recirculation along with the mean equivalent potential temperature of its poleward and equatorward components are used to recover an approximate overturning circulation on moist isentropes from which it is shown that the moist recirculation accounts for the difference between the meridional circulation averaged on dry and on moist isentropes.
\end{abstract}

\section{Introduction}

In midlatitudes, the meridional circulation is dominated by baroclinic eddies that convert available potential energy from a baroclinically unstable background state into kinetic energy. Although these eddies are responsible for

\footnotetext{
* Supplemental information related to this paper is available at the Journals Online Web site: http://dx.doi.org/10.1175/JAS-D-11-0124.s1.

Corresponding author address: Frédéric Laliberté, Department of Physics, University of Toronto, 60 St. George Street, Toronto ON M5S 1A7, Canada.

E-mail: frederic.laliberte@utoronto.ca
}

transporting most of the momentum, heat, and moisture from the subtropics to higher latitudes, their transient nature makes their climatology difficult to describe [Lorenz 1967; see Schneider (2006) for a review]. It thus remains a challenge to quantify the intensity and thermodynamic structure of these ubiquitous eddies.

The dry isentropic circulation (Gallimore and Johnson 1981; Johnson 1989; Karoly et al. 1997; Held and Schneider 1999) describes the mean meridional circulation by accounting for the midlatitude eddies' sensible heat transport. The dry isentropic circulation accounts for the sensible heat transport by separating warm, poleward flowing air with high dry potential temperatures and cold, equatorward flowing air with low dry potential temperatures. 
Furthermore, since the eddy time scale is short compared to the radiative damping time scale, dry potential temperature can be viewed as a material invariant-as long as latent heat release is neglected. In this regard, the isentropic circulation can be viewed as a more accurate approximation of the mean Lagrangian trajectories of air parcels than the Eulerian-mean circulation.

Midlatitudes eddies, in addition to transporting sensible heat, also transport latent heat as part of the global hydrological cycle. Moisture is extracted from the subtropical planetary boundary layer (Yang and Pierrehumbert 1994; Pierrehumbert 1998) and subsequently transported poleward and upward. The latent heat released during the accompanying phase transitions has a great influence on the eddies' total energy transport and accounts for a third to a half of the poleward energy transport in the storm tracks as described by Trenberth and Stepaniak (2003; see their Fig. 1). Schneider et al. (2006) calculated the eddy moisture transport on dry isentropic surfaces and showed that in the subtropics there exists a balance between the influx of moisture from the Hadley circulation and the poleward transport of moisture by extratropical eddies. They argued ${ }^{1}$ that the direct effect of moisture on subtropical eddies leads only to a small correction to their analysis and neglected it altogether. However, moist air is usually brought toward saturation as it is cooled through adiabatic ascent. Once saturated, the moist air either follows a slanted moist isentrope or ascends in deep convective towers within the storm tracks. Because of the associated phase transition, the dry potential temperature will be not conserved on the displaced parcels. This indicates an important limitation of using the dry isentropic average as a semi-Lagrangian analysis.

This limitation was highlighted in recent analyses by Czaja and Marshall (2006) and Pauluis et al. (2008, 2010), who accounted for the effect of latent heat release on the atmospheric circulation by analyzing the circulation on moist isentropes, defined as surfaces of constant equivalent potential temperature. They have compared the circulations on dry and moist isentropes and shown that the latter is significantly stronger in the midlatitudes. Unlike the circulation on dry isentropes, the circulation on moist isentropes includes the total heat flux-sensible plus latent. Moreover, while the dry potential temperature is not conserved for adiabatic saturated motions, the equivalent potential temperature is [although it is not exactly conserved when precipitation and freezing occur, as explained in the appendix of Pauluis et al. (2010)]. In regions where condensation occurs, it is therefore expected

\footnotetext{
${ }^{1}$ See the last paragraph of their section 2.
}

that the moist isentropic circulation captures Lagrangian trajectories more accurately than the dry isentropic circulation. The circulation on moist isentropes is indeed stronger in the subtropics and midlatitudes where latent heat release by condensation is a key diabatic heating source (Pauluis et al. 2008).

Pauluis et al. (2010) showed that the difference in mass transport between dry and moist isentropic circulations is intimately tied to the eddy transport of water vapor and the ascent of warm, moist subtropical air within the storm tracks. In this paper, we further investigate the relationship between the eddy transport of water vapor and the enhanced mass transport in the moist isentropic circulation. In doing so, we aim at reconciling the "dry" description, such as that of Schneider et al. (2006), which considers an eddy transport of water vapor superimposed on the dry isentropic circulation, and the "moist" perspective of Pauluis et al. $(2008,2010)$ in which latent heat is directly included in the definition of the moist isentropes.

In section 2 we define the meridional mass flux joint isentropic distribution, which records the meridional mass fluxes according to their dry and equivalent potential temperatures simultaneously. In section 3, we describe a simplified decomposition of the joint distribution on dry potential temperature surfaces into two directional flows: northward and southward. Using this decomposition we calculate the mean moist stratification associated with each directional flow and use it to interpret which type of convection (slantwise vs upright) is responsible for the midlatitudes' crossisentropic transport of moisture. We further show how the directional averages can be related to the climatological mean and variance. In section 4 , we use the directional fluxes to create an approximate circulation on moist isentropes and compare it to its exact counterpart. The results are summarized and discussed in section 5 .

In this study, we use data obtained from the 40-yr European Centre for Medium-Range Weather Forecasting (ECMWF) Re-Analysis (ERA-40) dataset that cover the period from 1981 to 2000 (Uppala et al. 2005). Variables from the dataset lie on a $1.125^{\circ} \times 1.125^{\circ} \mathrm{reg}$ ular Gaussian grid at T106 spectral truncation for 60 hybrid model levels. The meridional velocity, air temperature and logarithm surface pressure were converted to the regular Gaussian grid from a T159 spectral representation and the specific humidity was converted from a reduced N80 Gaussian grid. ${ }^{2}$

\footnotetext{
${ }^{2}$ Produced by and available from the National Center for Atmospheric Research Data Support Section, dataset ds127.2.
} 


\section{Joint isentropic distribution and isentropic circulation}

The analyses performed in this paper are based on the meridional mass flux joint distribution defined as

$$
M\left(\phi, \theta^{\prime}, \theta_{e}^{\prime}\right)=2 \pi a \cos \phi\left[\int_{0}^{p_{\text {surf }}} v \delta\left(\theta^{\prime}-\theta\right) \delta\left(\theta_{e}^{\prime}-\theta_{e}\right) \frac{d p}{g}\right],
$$

where $[\cdot]$ represents a zonal and temporal mean, $\delta(\cdot)$ corresponds to the Dirac function, $p_{\text {surf }}$ is the surface pressure, $v$ is the meridional velocity, $\theta$ is the dry potential temperature, and $\theta_{e}$ is the equivalent potential temperature [our Eq. (1) corresponds to Eq. (1) in Pauluis et al. (2010)]. The remaining quantities are defined in Table 1 . The mass flux joint distribution records the meridional mass fluxes according to their dry $\theta^{\prime}$ and equivalent $\theta_{e}^{\prime}$ potential temperature values. For a given air parcel, the two potential temperatures differ only if the parcel is moist corresponding to a positive total water content $q_{T}$. In this case the two potential temperatures are related according to

$$
\theta_{e}^{\prime} \approx \theta^{\prime} \exp \left(\frac{L_{v 0}}{c_{p} T_{0}} q_{T}\right)
$$

Before computing the mass flux joint distribution we linearly interpolate the meridional velocity, specific humidity, and temperature onto 260 equally spaced $\sigma=$ $p / p_{\text {surf }}$ levels. The dry potential temperature and the equivalent potential temperature are then calculated using the interpolated pressure, air temperature, and specific humidity (Emanuel 1994). We assume that moist air is an ideal mixture of gases and make use of the Clausius-Clapeyron equation. The mass flux joint distribution (1) is then computed numerically by replacing the inner integral and delta functions with their discrete equivalents. We use a discrete delta function derived by Roma et al. (1999) that conserves the first moments of the distribution:

$\sum_{\theta^{\prime}} \sum_{\theta_{e}^{\prime}} \theta^{\prime} M\left(\phi, \theta^{\prime}, \theta_{e}^{\prime}\right) \Delta \theta^{2}=2 \pi a \cos \phi\left[\sum_{k=1}^{260} \theta_{k} v_{k}(\phi, \lambda, t) \frac{\Delta p_{k}}{g}\right]$

where $k$ refers to the interpolated vertical levels. A similar expression is obtained when we replace $\theta^{\prime}$ by $\theta_{e}^{\prime}$ in front of $M$ and $\theta_{k}$ by $\theta_{e k}$ in front of $v_{k}$. This numerical representation ensures that the resulting joint distribution accurately captures meridional fluxes of both potential temperatures. The target grid $\left(\theta^{\prime}, \theta_{e}^{\prime}\right)$ spacing is uniform and corresponds to $\Delta \theta=1 \mathrm{~K}$.
TABLE 1. List of symbols.

\begin{tabular}{ll}
\hline \hline$\lambda, \phi, p, t$ & Longitude, latitude, pressure, time \\
$g, a$ & Gravitational acceleration, earth's radius \\
{$[\cdot]$} & Zonal and temporal average: function of $\phi$ \\
$\langle\cdot\rangle$ & Zonal and temporal average along lines of \\
& constant $\theta$ : function of $(\phi, \theta)$ \\
$\overline{(\cdot)^{*}}$ & Mass-weighted zonal and temporal average \\
& along lines of constant $\theta, \overline{(\cdot)^{*}=\langle\rho \cdot\rangle /\langle\rho\rangle:}$ \\
& function of $(\phi, \theta)$ \\
$\langle\cdot\rangle^{ \pm}$ & Directional isentropic mass flux-weighted average \\
$\langle\rho v\rangle^{ \pm}$ & along lines of constant $\theta$ : functions of $(\phi, \theta)$ \\
$M$ & Directional mass fluxes: functions of $(\phi, \theta)$ \\
$H(\cdot)$ & Mass flux joint distribution: function of $\left(\phi, \theta, \theta_{e}\right)$ \\
& Heaviside function; 0 for a negative argument and \\
$\theta, \theta_{e}$ & 1 otherwise \\
& Dry potential temperature, equivalent \\
$L_{v 0}$ & potential temperature \\
$c_{p}$ & Latent heat of vaporization at $0^{\circ} \mathrm{C}$ \\
$T_{0}$ & Specific heat of air \\
\hline & Reference temperature $\left(0^{\circ} \mathrm{C}\right)$
\end{tabular}

Using the mass flux joint distribution, one can recover the dry isentropic streamfunction $\Psi_{\theta}\left(\phi, \theta^{\prime}\right)$, which quantifies the net mass flux below the isentropic surface with dry potential temperature $\theta^{\prime}$. It can either be calculated directly from the meridional velocity or from the mass flux joint distribution as a projection onto dry isentropes; that is,

$$
\begin{aligned}
\Psi_{\theta}\left(\phi, \theta^{\prime}\right) & =2 \pi a \cos \phi\left[\int_{0}^{p_{\text {surf }}} v H\left(\theta^{\prime}-\theta\right) \frac{d p}{g}\right] \\
& =\int_{0}^{\theta^{\prime}} \int_{0}^{\infty} M\left(\phi, \theta, \theta_{e}\right) d \theta_{e} d \theta,
\end{aligned}
$$

where $H(\cdot)$ is the Heaviside function. The study of the dry isentropic streamfunction thus gives us a means to understand the mass flux joint distribution.

Figure 1 shows the dry isentropic streamfunction during December-February (DJF) and June-August (JJA). In the winter hemisphere, the circulation is a single equator-to-pole cell, with high dry potential temperature air flowing poleward and lower dry potential temperature air flowing equatorward. In the tropics, a well-defined Hadley cell exists and it connects to a direct cell in midlatitudes. The Hadley cell's strength does not differ substantially from its strength in the Eulerian-mean circulation as both circulations have maxima on the order of $200 \mathrm{~Sv}^{3}$ The extratropical circulation, which peaks around $40^{\circ}$ latitude (both seasons and both hemispheres), is weaker than the tropical circulation. During the Northern Hemisphere $(\mathrm{NH})$ summer, the circulation is very weak

\footnotetext{
${ }^{3}$ We use Sverdrup units to quantify mass fluxes: $1 \mathrm{~Sv}=10^{9}$ $\mathrm{kg} \mathrm{s}^{-1}$. This definition corresponds to the mass flux produced by a $1-\mathrm{Sv}$ flow of water with density $10^{3} \mathrm{~kg} \mathrm{~m}^{-3}$.
} 

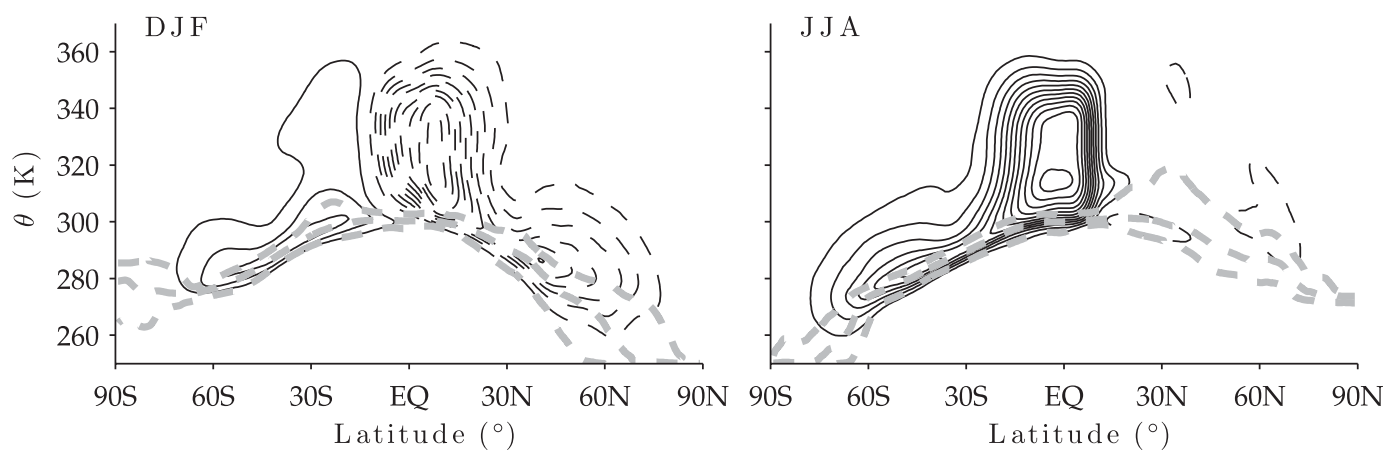

FIG. 1. The dry isentropic circulation (2) during (left) DJF and (right) JJA. Solid contours are positive and dashed contours are negative. The contours range from 20 to $240 \mathrm{~Sv}$ in $20-\mathrm{Sv}$ increments. The three gray dashed lines correspond to the 10th, 50th, and 90th percentiles of the dry potential temperature surface distribution.

and the dry streamfunction essentially vanishes for this season. In each panel, the gray dashed lines indicate the 10th, 50th, and 90th percentiles of the surface dry potential temperature distribution. The equatorward branch of the circulation in midlatitudes occurs over a narrow range of dry potential temperatures that are typical of surface values as noted by Held and Schneider (1999). On the other hand, the poleward branch of the circulation takes place over a wider range of dry potential temperatures corresponding to midtropospheric dry potential temperature values.

The net meridional mass flux on dry isentropic surfaces can be obtained by either taking a vertical derivative of the streamfunction (2) or by integrating the mass flux joint distribution over $\theta_{e}$; for example,

$$
\langle\rho v\rangle=\partial_{\theta^{\prime}} \Psi_{\theta}\left(\phi, \theta^{\prime}\right)=\int_{0}^{\infty} M\left(\phi, \theta^{\prime}, \theta_{e}\right) d \theta_{e} .
$$

The net mass flux on dry isentropic surfaces at $35^{\circ} \mathrm{N}$ during DJF is shown in the left panel of Fig. 2 (dot-dashed curve). It is southward (i.e., negative) below $290 \mathrm{~K}$, with a minimum of $-12 \mathrm{~Sv} \mathrm{~K}^{-1}$ at $\theta^{\prime}=285 \mathrm{~K}$, and northward (i.e., positive) above $290 \mathrm{~K}$, with a maximum of $7.5 \mathrm{~Sv} \mathrm{~K}^{-1}$ at $\theta^{\prime}=293 \mathrm{~K}$. For the remainder of this paper and in order to simplify our notation we drop the primes when referring to the phase-space dry and equivalent potential temperatures.

While the circulation on dry isentropes provides a more complete description of the meridional circulation than the Eulerian-mean circulation, it still does not fully describe the hydrological cycle associated with the overturning circulation. As already mentioned, Schneider et al. (2006) showed that there is a nontrivial eddy flux of water vapor on dry isentropes, implying an important poleward transport of latent heat that is not included in the circulation on dry isentropes. The existence of such a transport is not surprising since poleward-moving air, which originates either from the subtropical regions or from the planetary boundary layer, has to carry more moisture than its equatorward-moving counterpart, which originates from the midtroposphere at higher latitudes. The moisture flux in the dry isentropic circulation should thus be understood as the result of opposing flows with the same dry potential temperature but with different moisture content, which translates into different equivalent potential temperatures.

These opposing air masses cancel out in a dry isentropic average but appear as distinct transports in the mass flux joint distribution, shown in the right panel of Fig. 2 for $\phi=35^{\circ} \mathrm{N}$ during NH DJF. ${ }^{4}$ This figure is similar to Fig. $3 d$ of Pauluis et al. (2010). The discussion about the white curves superimposed on top of the joint distribution is postponed until section 3c. On every dry isentrope above $\theta=289 \mathrm{~K}$, indicated as a dotted line spanning the two panels, there are both positive (solid) and negative (dashed) contours. Since the procedure to compute net mass fluxes on dry isentropes from the joint distribution involves the integration across moist isentropes, as in Eq. (3), important cancellations between the northward (positive) and southward (negative) flows will occur on dry isentropes above $289 \mathrm{~K}$. In the next section, we isolate the equatorward and poleward transports using the mass flux joint distribution in order to better understand the role of moisture in the general circulation.

\section{Directional mass fluxes and water vapor transport}

\section{a. Directional mass fluxes}

The cancellations observed in the previous section can be avoided if the mass flux joint distribution is separated

\footnotetext{
${ }^{4}$ The mass flux joint distribution for a selection of other latitudes is provided as online supplemental material.
} 

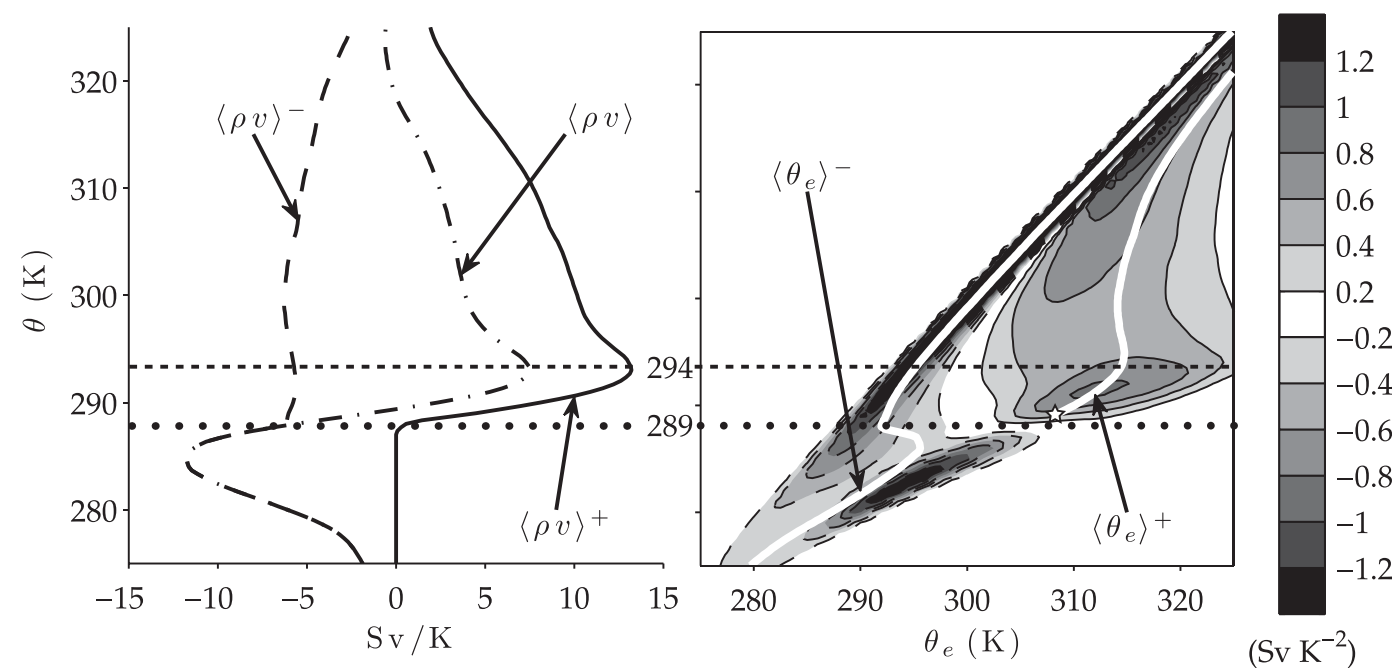

FIG. 2. DJF at $\phi=35^{\circ} \mathrm{N}$. (left) Net mass transport $\langle\rho v\rangle$ (dot-dashed), net equatorward mass flux $\langle\rho v\rangle^{-}$(dashed), and poleward mass flux $\langle\rho v\rangle^{+}$(solid). (right) Meridional mass flux joint distribution at $\phi=35^{\circ} \mathrm{N}$ during DJF. The solid contours represent poleward mass fluxes and the dashed contours correspond to equatorward transports. The white curves labeled $\left\langle\theta_{e}\right\rangle^{-}$and $\left\langle\theta_{e}\right\rangle^{+}$identify the mean $\theta_{e}$ on a dry potential temperature surface in the equatorward and poleward mass fluxes, respectively. These are discussed in section $3 \mathrm{c}$.

into northward, $M^{+}\left(\phi, \theta, \theta_{e}\right)$, and southward, $M^{-}(\phi$, $\left.\theta, \theta_{e}\right)$, components defined as

$$
\begin{aligned}
& M^{+}\left(\phi, \theta, \theta_{e}\right)=\max \left[M\left(\phi, \theta, \theta_{e}\right), 0\right] \text { and } \\
& M^{-}\left(\phi, \theta, \theta_{e}\right)=\min \left[M\left(\phi, \theta, \theta_{e}\right), 0\right],
\end{aligned}
$$

and indicated in the right panel of Fig. 2 by dashed and solid contours, respectively. Note that this directionally dependent decomposition of the joint distribution does not discard any information. The net mass flux can be recovered by adding the components: $M\left(\phi, \theta, \theta_{e}\right)=$ $M^{+}\left(\phi, \theta, \theta_{e}\right)+M^{-}\left(\phi, \theta, \theta_{e}\right)$. This decomposition is advantageous because the individual southward and northward contributions are sign-definite, and each one of them can be considered as a standard two-dimensional distribution at every latitude.

The net mass transport on dry isentropes can also be decomposed into northward $\langle\rho v\rangle^{+}$and southward $\langle\rho v\rangle^{-}$ components:

$$
\begin{aligned}
& \langle\rho v\rangle^{+}=\int_{0}^{\infty} M^{+}\left(\phi, \theta, \theta_{e}\right) d \theta_{e}, \\
& \langle\rho v\rangle^{-}=\int_{0}^{\infty} M^{-}\left(\phi, \theta, \theta_{e}\right) d \theta_{e} .
\end{aligned}
$$

The net northward and southward directional fluxes at $35^{\circ} \mathrm{N}$ DJF are shown in the left panel of Fig. 2 as solid and dashed curves, respectively.
The full latitudinal structure of the directional fluxes during DJF and JJA are shown in the top panels of Figs. 3 and 4, respectively. The top left panels show northward directional fluxes $\langle\rho v\rangle^{+}$and the top right panels show southward directional fluxes $\langle\rho v\rangle^{-}$. The net mass flux on dry potential temperature surfaces $\langle\rho v\rangle$ is shown in bottom right panels. In those panels, the gray dashed curve corresponds to the median surface dry potential temperature. These cancellations can be quantified according to

$$
\langle\rho v\rangle^{\prime}=\min \left(\langle\rho v\rangle^{+},-\langle\rho v\rangle^{-}\right)
$$

and are shown in the bottom left panels of Figs. 3 and 4. The physical significance of this quantity is addressed later in section $3 b$. Since the polar regions are outside the scope of this study, we restrict our analysis from $60^{\circ} \mathrm{S}$ to $60^{\circ} \mathrm{N}$ for Figs. 3-6. Polar regions have very weak net mass fluxes, which makes comparisons with lower latitudes difficult.

The bottom right panels of Figs. 3 and 4 correspond to net mass fluxes on dry potential temperature surfaces, $\langle\rho v\rangle$, and are equal-through Eq. (3) - to the vertical derivative of the dry isentropic circulation shown in Fig. 1. One can identify the Hadley cell by the strong poleward mass fluxes at $\theta \approx 350 \mathrm{~K}$ and the strong equatorward surface fluxes at $\theta \approx 300 \mathrm{~K}$ between the equator and $30^{\circ}$ in the winter hemisphere. The tropical surface flow has a warm component coming from the winter hemisphere and a colder-and weaker-component coming from the summer hemisphere. In the winter hemispheres, 

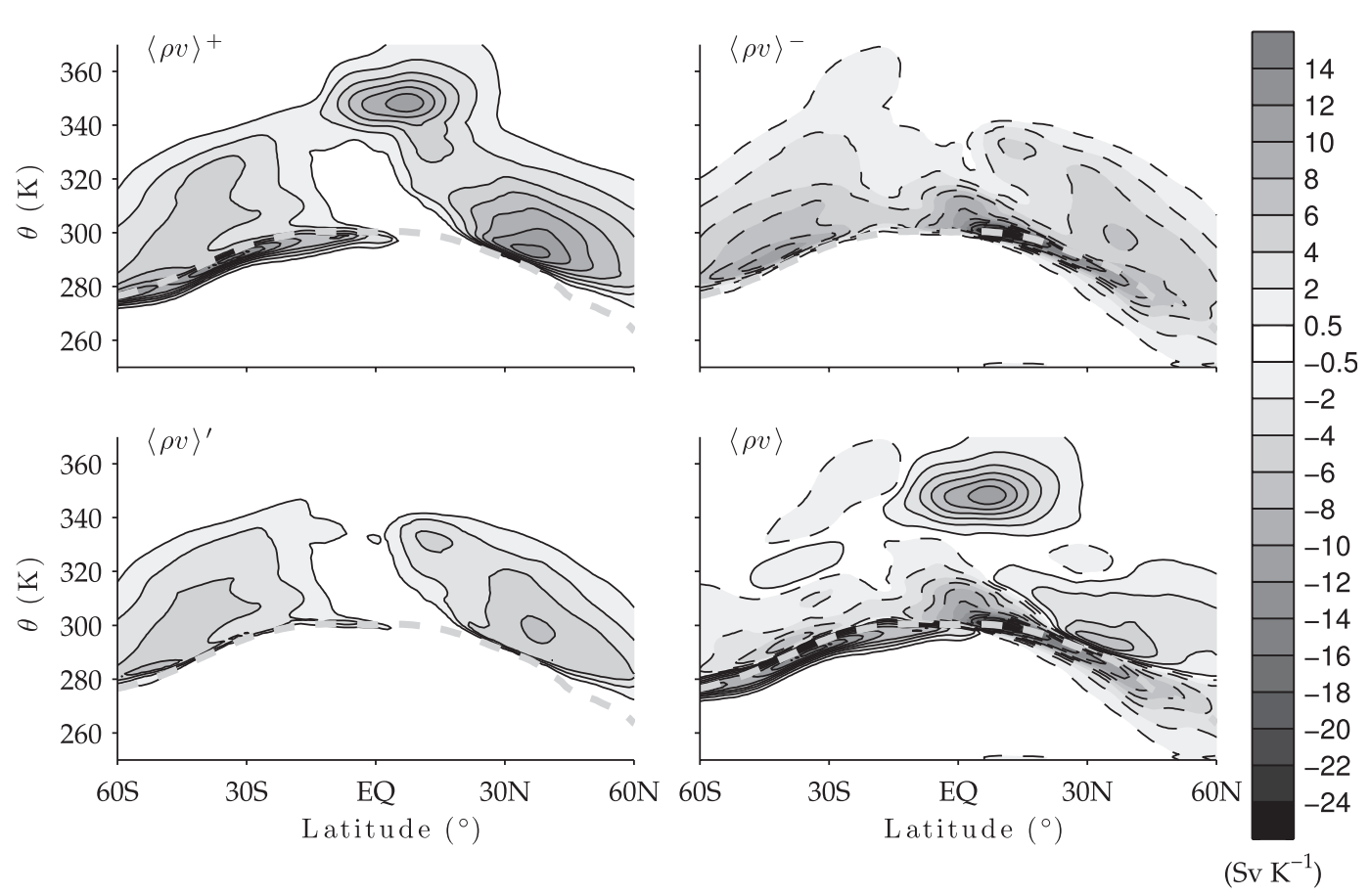

FIG. 3. Meridional mass fluxes on dry potential temperature surfaces during DJF. (top left) Northward mass fluxes. (top right) Southward mass fluxes. (bottom left) The moist recirculation $\langle\rho v\rangle^{\prime}$ [see Eq. (6)]. (bottom right) Net mass fluxes. Solid contours are positive and dashed contours are negative. The gray dotted line shows the median surface dry potential temperature. Note that the contouring is linear except for the lowest contour.

the midlatitudes' equatorward flow is intense and occurs within a narrow range of dry potential temperatures mostly below the median surface dry potential temperature [see Held and Schneider (1999) for a detailed analysis] that merges with the surface flow of the Hadley cell in the subtropics. The poleward flow is weaker and spread over a broad range of dry potential temperatures from surface values to approximately $300-310 \mathrm{~K}$. The midtropospheric poleward flow becomes important in the extratropics and is disconnected from the poleward flow of the Hadley cell by small patches of weak midtropospheric equatorward fluxes. This separation between the poleward flows in the tropics and midlatitudes disappears in the poleward directional fluxes (top left panel of Fig. 3 and top right panel of Fig. 4). The poleward directional fluxes in the Hadley cell are now clearly connected to those in midlatitudes by mass fluxes that are about a third to a half of the mass flux strength in the poleward net fluxes (bottom right panels of Figs. 3 and 4). Such a connection has been noted in previous studies (Pierrehumbert 1998; Schneider et al. 2006) and is related to the eddies extracting moisture from the subtropics. The fact that the net mass fluxes on dry isentropes $\langle\rho v\rangle$ vanish in this region suggests that there are corresponding equatorward directional fluxes that cancel the poleward directional fluxes.
The top right panels of Figs. 3 and 4 show the corresponding equatorward directional fluxes during DJF and JJA, respectively. During DJF, the subtropical equatorward directional fluxes in the NH span dry potential temperature values from 320 to $340 \mathrm{~K}$. During JJA, the subtropical fluxes in the Southern Hemisphere (SH) span values from 320 to $330 \mathrm{~K}$. The equatorward directional fluxes in midlatitudes during both seasons and both hemispheres are distributed across the whole midtroposphere and have a much broader $\theta$ extent than the corresponding equatorward flux contribution to the net mass flux $\langle\rho v\rangle$ (see dashed contours in bottom right panels of Figs. 3 and 4). While these midtropospheric mass fluxes are not as intense as the surface equatorward fluxes, they are spread from surface dry potential temperatures values up to $320 \mathrm{~K}$ and occur poleward of $20^{\circ}$. A significant portion of the midtropospheric equatorward mass fluxes are cancelled by poleward directional fluxes (cf. top panels of Figs. 3 and 4). This cancellation can be inferred from the noticeable absence of net mass fluxes between $20^{\circ}$ and $40^{\circ} \mathrm{N}$ and 310 and $330 \mathrm{~K}$ in the bottom right panel of Fig. 3 .

We have previously observed that the dry isentropic circulation was weak during the $\mathrm{NH}$ summer, shown in the right panel of Fig. 1. The net mass fluxes, in the bottom right panel of Fig. 4, are weak during that season 

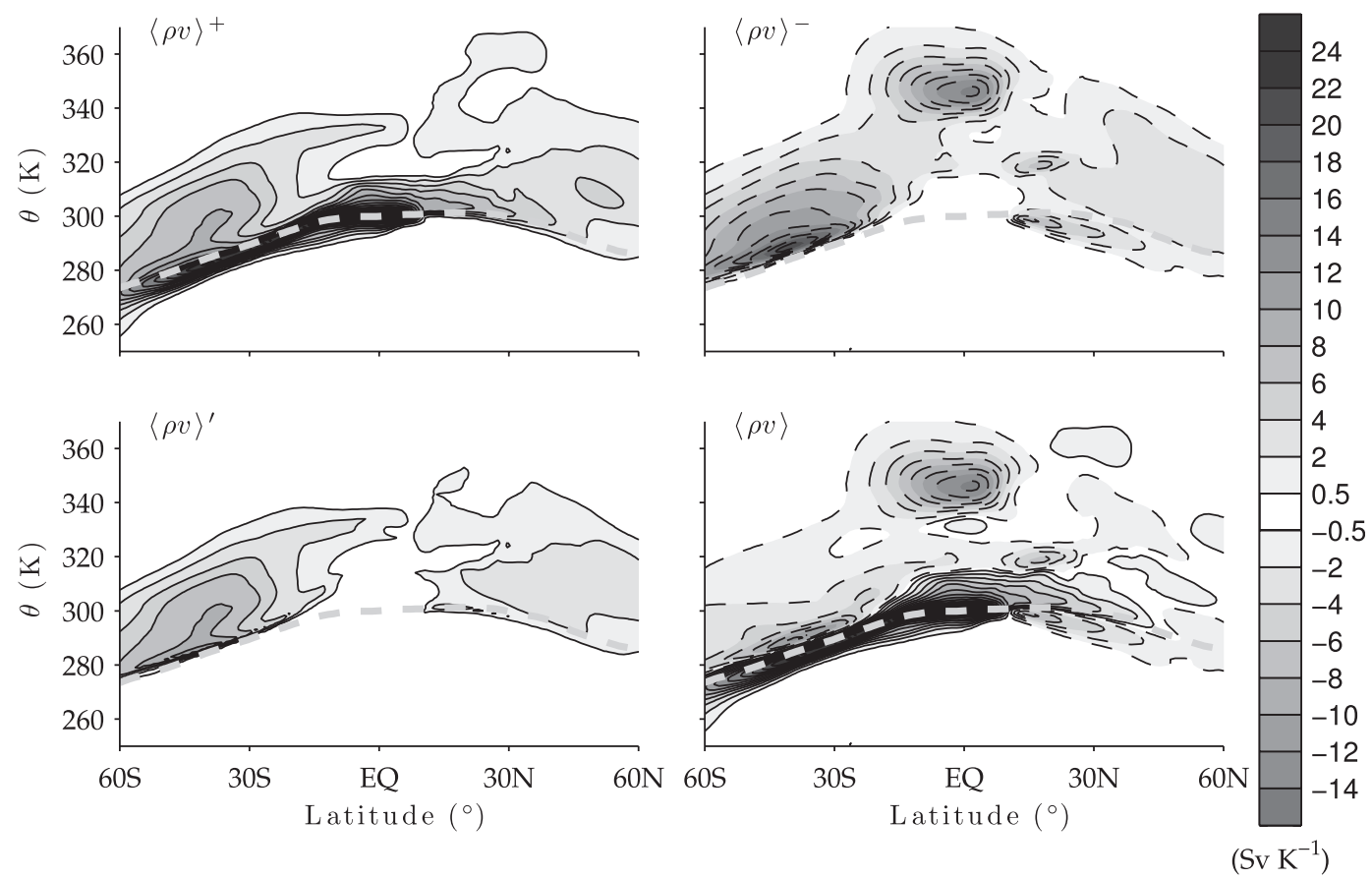

FIG. 4. As in Fig. 3, but for JJA.

and are very weak in comparison to the $\mathrm{NH}$ winter net mass fluxes. If we compare the $\mathrm{NH}$ fluxes in the top panels in Figs. 3 and 4, we observe that the directional mass fluxes are also weak relative to those during $\mathrm{NH}$ winter. Indeed, over the region from $30^{\circ}$ to $60^{\circ} \mathrm{N}$ the poleward directional fluxes $\langle\rho v\rangle^{+}$during DJF are about 3 times stronger than those during JJA, which can be verified by contrasting the top left panel of Fig. 3 against Fig. 4. However, the poleward fluxes are still significant and yet the net poleward fluxes $\langle\rho v\rangle$ in the $\mathrm{NH}$ essentially vanish during JJA. This means that on a given dry isentrope during JJA the moist poleward component mostly cancels the drier equatorward component, leading to a net poleward transport of latent heat with a vanishing sensible heat transport. A similar, less pronounced, cancellation occurs in both hemispheres and during both seasons.

\section{b. Moist recirculation}

The directional mass fluxes $\langle\rho v\rangle^{+}$and $\langle\rho v\rangle^{-}$occur on the same dry potential temperature surface but at different values of equivalent potential temperature. This difference in equivalent potential temperature indicates that the two directional mass fluxes describe thermodynamically different air parcels moving in opposite directions. The net poleward mass flux is thus the result of a cancellation between the two directional fluxes and is therefore significantly weaker than either directional flux. In the midtroposphere, the net mass fluxes are poleward and the magnitude of the mass flux cancellation will be equal to the magnitude of the equatorward directional fluxes. More generally, the cancellation $\langle\rho v\rangle^{\prime}$ between the two directional fluxes is equal to the smallest flux in absolute value, as shown previously in Eq. (6). These cancellations correspond to the portion of the net poleward mass fluxes in moist regions that is exactly balanced by an equatorward transport of mass in dry regions.

The cancellations $\langle\rho v\rangle^{\prime}$ shown in the bottom panels of Figs. 3 and 4 represent two fluxes of equal strength going in opposite direction superimposed at each point of the $(\phi, \theta)$ plane. The first flux is poleward and moist while the second is equatorward and dry. This recirculating flow is hidden within the dry circulation because it transports latent heat without transporting any sensible heat. It thus represents the purely moist part of the meridional overturning circulation. Because of this interpretation, we call the cancellations $\langle\rho v\rangle^{\prime}$ the moist recirculation (MR). As seen in Figs. 3 and 4, the majority of the MR is located above the surface median dry potential temperature in midlatitudes between $30^{\circ}$ and $60^{\circ}$. Its extent in $\theta$ is similar to the corresponding extent of the midlatitudes directional fluxes and its strength is comparable to that of equatorward directional fluxes.

The MR in the winter hemisphere extends deep into the subtropics, as low as $15^{\circ}$, attaining its peak in the 


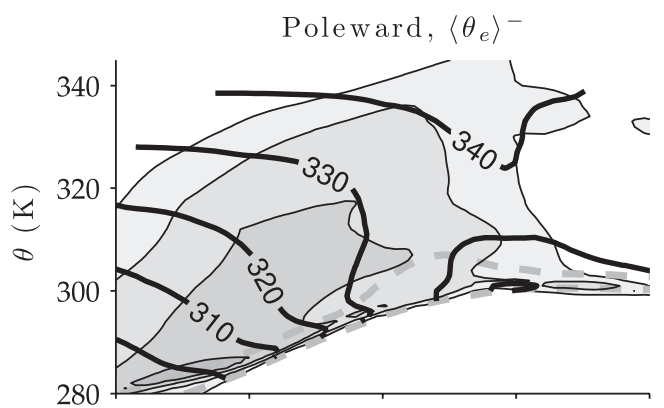

Equatorward, $\left\langle\theta_{e}\right\rangle^{+}$

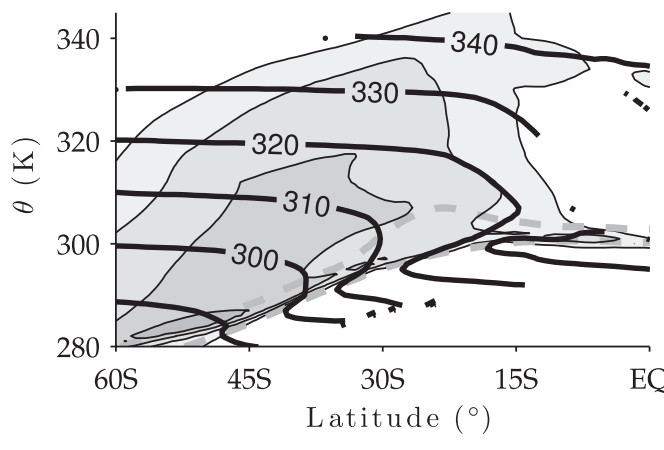

Poleward, $\left\langle\theta_{e}\right\rangle^{+}$

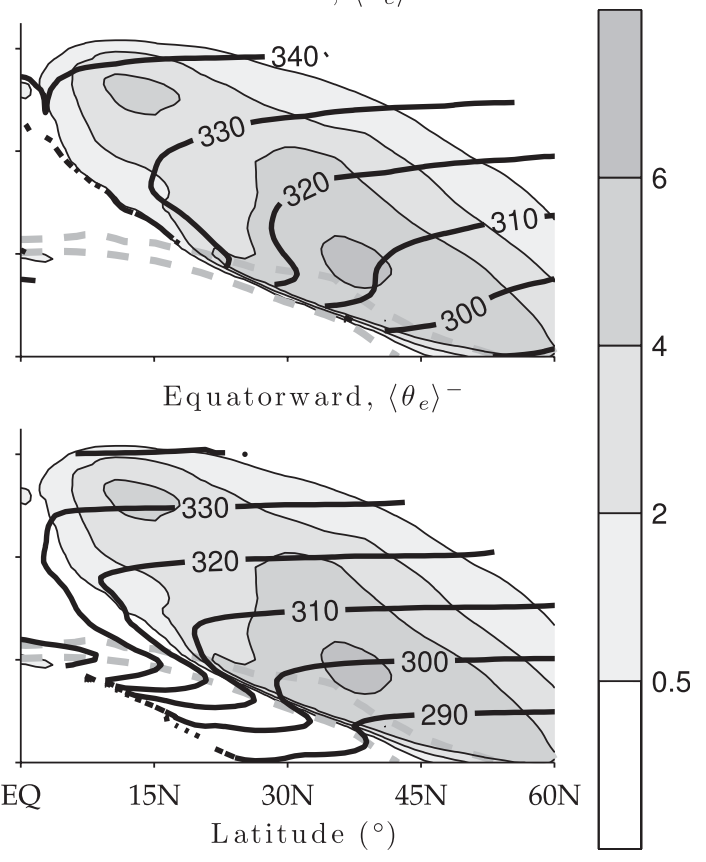

$\left.(\mathrm{Sv} \mathrm{K})^{-1}\right)$

FIG. 5. Mean equivalent potential temperature in directional fluxes overlaid on (top) poleward and (bottom) equatorward directional fluxes during DJF: (top) (left) $\left\langle\theta_{e}\right\rangle^{-}$and (right) $\left\langle\theta_{e}\right\rangle^{+}$; and (bottom) (left) $\left\langle\theta_{e}\right\rangle^{+}$and (right) $\left\langle\theta_{e}\right\rangle^{-}$. Shaded contours show the moist recirculation as in Figs. 3 (lower left). Note that the contouring is linear except for the lowest contour. The gray dashed curves correspond to the 50th and 90th percentiles of the surface dry potential temperature distribution.

mid- to upper troposphere at $\theta \approx 330 \mathrm{~K}$ and vanishing for dry potential temperatures close to surface values. This peak corresponds to the lower portion of the poleward mass fluxes in the Hadley circulation and it suggests that the MR is responsible for the connection between the subtropics and midlatitudes. During winter in midlatitudes, the MR exhibits intense mass fluxes at $37^{\circ}$ and $\theta \approx 300 \mathrm{~K}$, of a magnitude of $3 \mathrm{~Sv} \mathrm{~K}^{-1}$ during DJF and $4 \mathrm{~Sv} \mathrm{~K}^{-1}$ during JJA. During SH winter, there are even larger mass fluxes in the lower troposphere. These are, however, of a different nature as they are for the most part related to surface fluxes.

The MR quantifies mass fluxes that can only be resolved using a moist isentropic average and it can therefore be used to understand the large difference between the dry and moist isentropic circulations during JJA in the NH (Pauluis et al. 2008). Indeed, in the NH and during JJA the MR mass fluxes have about one-third to one-half of their DJF value (cf. top left panels of Figs. 3 and 4). This implies that the vanishing dry isentropic circulation during the $\mathrm{NH}$ summer is misleading because it is in large part the result of cancellations between opposite branches of the MR and not the result of an eddy activity that is much weaker than during the $\mathrm{NH}$ winter.

\section{c. Thermodynamics and moist ascent}

The MR can only exist if there is a difference of equivalent potential temperature-or moisture contentbetween poleward and equatorward mass fluxes on a dry isentrope. To recover the mean equivalent potential temperatures transported by the directional fluxes, one can use the northward and southward mass flux joint distributions (4):

$$
\begin{aligned}
& \left\langle\theta_{e}\right\rangle^{+}=\int_{0}^{\infty} \theta_{e} M^{+}\left(\phi, \theta, \theta_{e}\right) d \theta_{e} /\langle\rho v\rangle^{+}, \\
& \left\langle\theta_{e}\right\rangle^{-}=\int_{0}^{\infty} \theta_{e} M^{-}\left(\phi, \theta, \theta_{e}\right) d \theta_{e} /\langle\rho v\rangle^{-} .
\end{aligned}
$$

The right panel of Fig. 2 shows these mean equivalent potential temperatures as thick white curves superimposed on the mass flux joint distribution at $35^{\circ} \mathrm{N}$ during DJF. The mean equivalent potential temperature of the southward-in this case equatorward-surface fluxes has a $\theta$ value corresponding to surface temperature and with a $\left\langle\theta_{e}\right\rangle^{-}$value consistent with high relative humidity. This equatorward flow, associated with eddies in midlatitudes, connects with the surface branch of the Hadley cell in the 


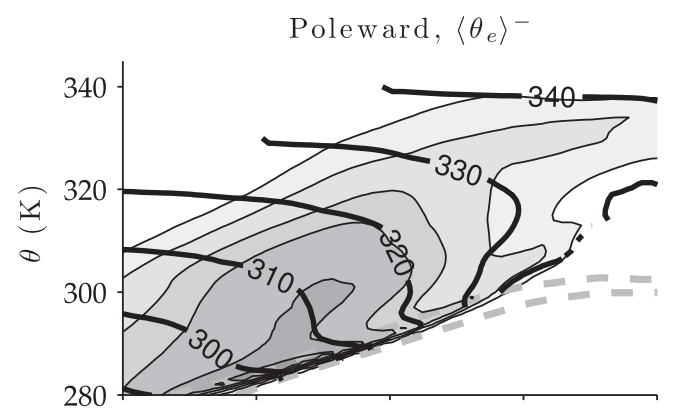

Equatorward, $\left\langle\theta_{e}\right\rangle^{+}$

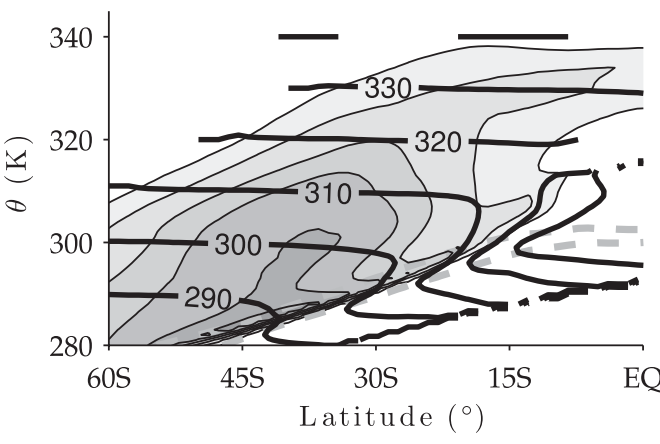

FIG. 6. As in Fig. 5, but during JJA.
Poleward, $\left\langle\theta_{e}\right\rangle^{+}$

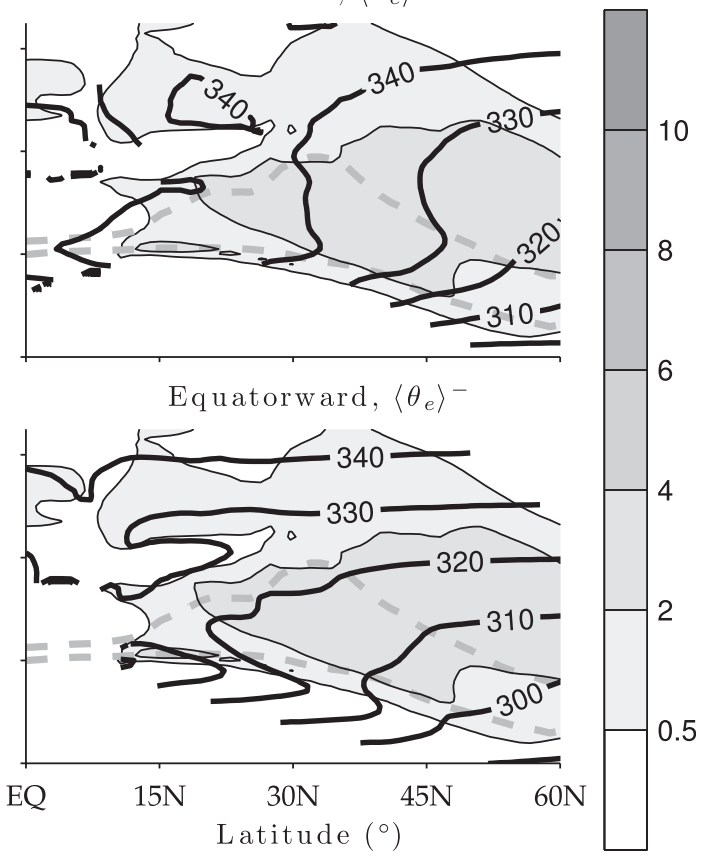

$\left(\mathrm{Sv} \mathrm{K}{ }^{-1}\right)$ subtropics. Moving along the $\left\langle\theta_{e}\right\rangle^{-}$curve, one reaches at $\theta=289 \mathrm{~K}$ a sharp transition from a moist $\left\langle\theta_{e}\right\rangle^{-}$value to a dry value-that is, along the line $\left\langle\theta_{e}\right\rangle^{-}=\theta$. This occurs approximately at the intersection with the dotted line, a line that was previously used to indicate the dry isentrope above which the poleward fluxes are nonzero. Above this sharp transition, $\left\langle\theta_{e}\right\rangle^{-}$remains along the main diagonal, therefore corresponding to dry air.

The average equivalent potential temperature of the northward-in this case poleward-flux becomes impor$\operatorname{tant}$ at $\theta=290 \mathrm{~K}$ with a value of $\left\langle\theta_{e}\right\rangle^{+} \approx 310 \mathrm{~K}$, a point we have indicated by a star. It then increases slightly with increasing $\theta$ until it peaks to $\left\langle\theta_{e}\right\rangle^{+}=315 \mathrm{~K}$ at $\theta=294 \mathrm{~K}$, roughly the level at which the northward fluxes attain their maximum, a fact that can be easily observed by following the thin dashed line spanning the two panels. The equivalent potential temperature then remains mostly constant until $\theta=305 \mathrm{~K}$, above which it increases with increasing $\theta$. Because $\left\langle\theta_{e}\right\rangle^{+}$overall varies little in the vertical, it indicates that the northward mass fluxes are thermodynamically similar across the troposphere and thus suggests large-scale moist ascents in the northward flow.

The total $\theta_{e}$ flux on dry isentropic surfaces can be recovered from the directional fluxes according to

$$
\left\langle\theta_{e} \rho v\right\rangle=\left\langle\theta_{e}\right\rangle^{+}\langle\rho v\rangle^{+}+\left\langle\theta_{e}\right\rangle^{-}\langle\rho v\rangle^{-} .
$$

The decomposition into directional fluxes and directional equivalent potential temperature thus accurately represents net fluxes of equivalent potential temperature. When the net mass fluxes on dry isentropes vanish Eq. (8) can be thought of as an eddy flux that is driven by a mass flux $\langle\rho v\rangle^{\prime}$ transporting $\theta_{e}$ proportional to the difference $\left\langle\theta_{e}\right\rangle^{+}-\left\langle\theta_{e}\right\rangle^{-}$. Recall that during NH JJA the net mass fluxes on dry isentropes essentially vanish and therefore the $\theta_{e}$ flux on the left-hand side of Eq. (8) will be the mass-weighted zonal-mean eddy flux of $\theta_{e}$ on dry isentropes and the right-hand side will be equal to $\left(\left\langle\theta_{e}\right\rangle^{+}-\left\langle\theta_{e}\right\rangle^{-}\right)\langle\rho v\rangle^{\prime}$.

In the $\mathrm{SH}$ and in the $\mathrm{NH}$ during DJF, the net mass fluxes do not vanish and therefore the MR cannot be exactly interpreted as an eddy mass flux. It is nevertheless convenient to assume that the opposing mass fluxes of the MR have a typical equivalent potential temperature equal to their respective directional $\theta_{e}$ : northward mass fluxes occur at $\left\langle\theta_{e}\right\rangle^{+}$and southward fluxes at $\left\langle\theta_{e}\right\rangle^{-}$. Under this assumption, meridional fluxes of $\theta_{e}$ associated with the northward branch of the MR will be equal to $\left\langle\theta_{e}\right\rangle^{+}\langle\rho v\rangle^{\prime}$ and fluxes associated with the southward branch will be equal to $-\left\langle\theta_{e}\right\rangle^{-}\langle\rho v\rangle^{\prime}$.

Figures 5 and 6 show the mass flux-weighted mean equivalent potential temperature-that is, Eqs. (7)superimposed on the MR from bottom left panels of Figs. 3 and 4 for DJF and JJA, respectively. The directional $\theta_{e}$ 
are plotted according to their direction within each hemisphere (poleward or equatorward) instead of according to their absolute direction (northward or southward) in order to highlight the similarities between poleward flows and between equatorward flows. The difference between the two directional equivalent potential temperatures (not shown), $\left\langle\theta_{e}\right\rangle^{+}-\left\langle\theta_{e}\right\rangle^{-}$, being positive in the $\mathrm{NH}$ and negative in the $\mathrm{SH}$, confirms that the MR is associated with a poleward flux of $\theta_{e}$ on dry isentropes. The MR thus represents a poleward latent heat transport since a difference of $\theta_{e}$ on a fixed dry isentrope is proportional to a difference in latent heat,

$$
\left\langle\theta_{e}\right\rangle^{+}-\left\langle\theta_{e}\right\rangle^{-} \sim \frac{L_{v 0}}{c_{\mathrm{pd}}} \Delta q_{T}
$$

where $\Delta q_{T}$ denotes the difference in specific humidity between the poleward and equatorward components. To simplify our description of Figs. 5 and 6, we will focus for the rest of this section on the NH DJF in the right panels of Fig. 5. Most of our observations carry over directly to both seasons of the SH. In NH JJA, our observations still hold but for much higher latitudes.

The directional $\theta_{e}$ profiles can be used to analyze the moist vertical stability profiles experienced by the equatorward and poleward fluxes. To demonstrate how, we look at the equatorward directional $\theta_{e}$ in bottom panel of Fig. 5. In midlatitudes, the equatorward $\theta_{e}$ profiles have large values of $\theta_{e}$ below the median surface $\theta$, associated with mass fluxes occurring within the planetary boundary layer. While these air masses contain sufficient latent heat to ascend to the midtroposphere and above, they are unlikely to do so because their equatorward motion mixes them with warmer boundary layer air masses from lower latitudes. Since on average this process warms equator bound air masses, these are therefore not brought closer to saturation, thus preventing their latent heat from being released and restricting their ability to ascend. Above the median surface dry potential temperature, the curves of constant equatorward $\theta_{e}$ are aligned with lines of constant $\theta$ and therefore depict dry equatorward flows.

The vertical structure of poleward $\theta_{e}$ in the top panel of Fig. 5 exhibits marked differences between the subtropics and midlatitudes. In the subtropics and up to $35^{\circ} \mathrm{N}$, the vertical profile exhibits a local minimum at $\theta \approx 310 \mathrm{~K}$, corresponding to midtropospheric values. The consequence of such a vertical profile can be better understood by considering a typical surface parcel at $29^{\circ} \mathrm{N}$ that lies on the $\left\langle\theta_{e}\right\rangle^{+}=320 \mathrm{~K}$ curve at $\theta=295 \mathrm{~K}$. At the beginning of its poleward motion, it follows a mostly horizontal (i.e., dry isentropic) path until $32^{\circ} \mathrm{N}$. If this parcel keeps moving poleward while preserving its

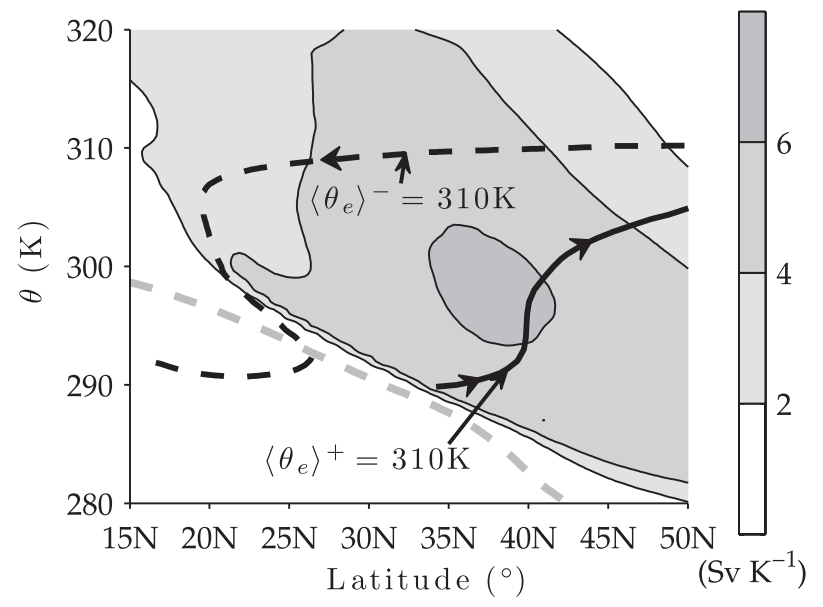

FIG. 7. A zoomed-in version of the top right panel of Fig. 5. The thick solid curve indicates $\left\langle\theta_{e}\right\rangle^{+}=310 \mathrm{~K}$ and the thick dashed curve indicates $\left\langle\theta_{e}\right\rangle^{-}=310 \mathrm{~K}$. Arrows indicate the likely path followed by the flow. The shaded contours indicate the moist recirculation. The gray dashed line represents the median of the surface dry potential temperature distribution.

$\left\langle\theta_{e}\right\rangle^{+}$, it will do so at a higher $\theta$ value-about $310 \mathrm{~K}-$ because no poleward mass fluxes have $\left\langle\theta_{e}\right\rangle^{+}=320 \mathrm{~K}$ below this level. Such a discontinuous path suggests a fast adjustment, interpreted as being most likely accomplished through deep upright convection. We cannot, however, rule out that other convective adjustment mechanisms can be consistent with the observed $\theta_{e}$ profile.

Poleward of $35^{\circ} \mathrm{N}$ in the top panel of Fig. 5, poleward $\theta_{e}$ profiles are increasing with increasing $\theta$. If we consider a parcel with typical surface $\theta$ moving poleward in this region, the issue of a discontinuous path does not arise, which contrasts with the situation in the subtropics. As a consequence, a test parcel at $40^{\circ} \mathrm{N}, \theta=$ $290 \mathrm{~K}$ on the curve $\left\langle\theta_{e}\right\rangle^{+}=310 \mathrm{~K}$ will follow the adiabatic path corresponding to the $\left\langle\theta_{e}\right\rangle^{+}=310 \mathrm{~K}$ curve all the way to the pole. Such a smooth path is suggestive of large-scale moist ascents associated with slantwise convection. Our analysis, however, does not allow us to exclude that a collection of deep convective events could contribute substantially to the net moist ascents.

To better illustrate the resulting poleward and equatorward thermodynamics cycle in midlatitudes, we isolate the $\left\langle\theta_{e}\right\rangle^{+}=310 \mathrm{~K}$ and $\left\langle\theta_{e}\right\rangle^{-}=310 \mathrm{~K}$ curves from the right panels of Fig. 5 and zoom in to produce Fig. 7. From this composite diagram, we first consider typical surface air parcels that enter the poleward branch of the circulation at $35^{\circ} \mathrm{N}$. Over a normal eddy time scale of 6-7 days, eddy motions remain mostly adiabatic and thus we can assume that typical parcels will follow an adiabatic path. As long as it is unsaturated, its dry potential 

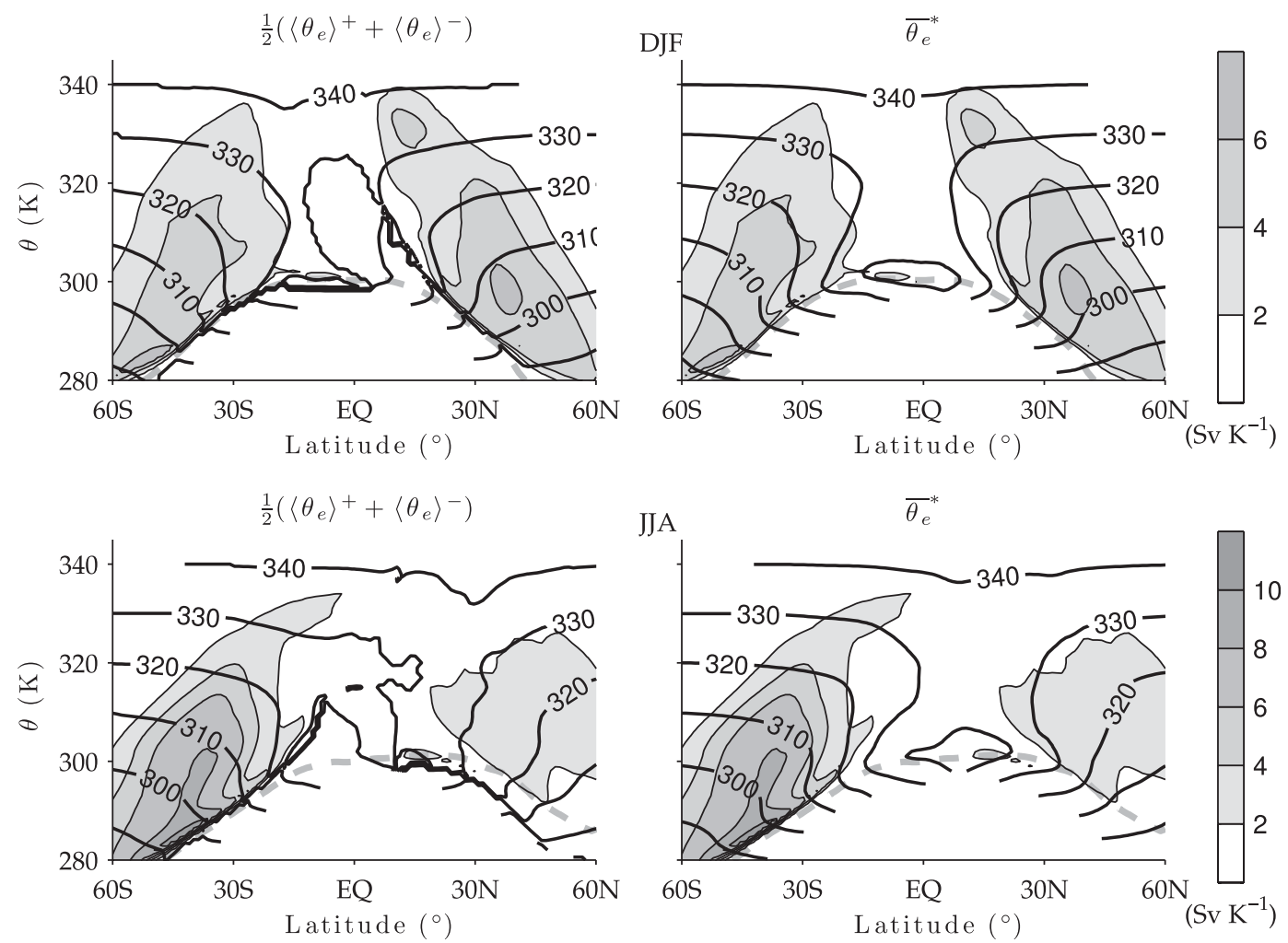

FIG. 8. A comparison between (left) the mean poleward and equatorward equivalent potential temperature $(1 / 2)\left(\left\langle\theta_{e}\right\rangle^{+}+\left\langle\theta_{e}\right\rangle^{-}\right)$and (right) the mass-weighted climatology $\bar{\theta}_{e}^{*}$, for (top) DJF and (bottom) JJA.

temperature and its equivalent potential temperature will be conserved. From $35^{\circ}$ to $40^{\circ} \mathrm{N}$, the $\left\langle\theta_{e}\right\rangle^{+}=$ $310 \mathrm{~K}$ curve that is representative of the equivalent potential temperature of typical parcels in the poleward flow is initially horizontal, which confirms that those parcels are unsaturated in the first leg of the MR cycle.

At around $40^{\circ} \mathrm{N}$, however, the $\left\langle\theta_{e}\right\rangle^{+}=310 \mathrm{~K}$ curve followed by typical parcels departs from the horizontal, which indicates a conversion of latent heat into specific heat. Those parcels in the poleward flow thus appear to undergo an upright moist ascent characterized by a vertical path from $\theta \approx 293 \mathrm{~K}$ to $\theta \approx 300 \mathrm{~K}$. After this transition, the $\left\langle\theta_{e}\right\rangle^{+}=310 \mathrm{~K}$ curve depicts a slanted moist isentropic trajectory that extends to the MR's upper and poleward edge, ultimately reaching the $\theta=$ $310 \mathrm{~K}$ isentrope. Typical returning parcels will move toward lower latitudes along the curve $\left\langle\theta_{e}\right\rangle^{-}=310 \mathrm{~K}$ : a horizontal line that is equal to the $\theta=310 \mathrm{~K}$ isentrope. If all the water in condensed liquid phase has been lost to precipitation in the poleward branch of the cycle, there will be no re-evaporation associated with this downward motion. The parcels' equivalent potential temperature will thus remain at $\left\langle\theta_{e}\right\rangle^{-}=310 \mathrm{~K}$ until it is remoistened in the boundary layer.
The portion of the $\left\langle\theta_{e}\right\rangle^{-}=310 \mathrm{~K}$ curve south of $25^{\circ} \mathrm{N}$ exhibits an "S" shape. Air parcels moving southward along the upper branch of the $\left\langle\theta_{e}\right\rangle^{-}=310 \mathrm{~K}$ are dry parcels displaced by the MR. Because the MR is weak in this region, we can assume that these air parcels are slower than earlier in the cycle and that their motions are more affected by radiative damping. As the parcels cool, they leave the $\left\langle\theta_{e}\right\rangle^{-}=310 \mathrm{~K}$ curve and enter the planetary boundary layer within the subtropics. In the boundary layer, they are mixed with near-surface air and subsequently moistened, which explains the high $\left\langle\theta_{e}\right\rangle^{-}$values close to the median surface dry potential temperature. Afterward, these air parcels continue equatorward and increase their moisture content. Because their $\theta$ value is unchanged during this process these air parcels will remain on average convectively stable until they reach the intertropical convergence zone.

\section{d. Relationship with climatological diagnostics}

In midlatitudes, the MR is stronger than net mass fluxes $\langle\rho v\rangle$, which implies that it dominates the circulation in that region. One can therefore assume that half of the time the troposphere is in a poleward-moving state 
and the other half of the time in an equatorward-moving state. If this assumption was valid it would imply that although the individual directional mean profiles are very different from the climatological mean they would still combine to generate the mean. We can test this assumption by verifying that the approximation

$$
\frac{1}{2}\left(\left\langle\theta_{e}\right\rangle^{+}+\left\langle\theta_{e}\right\rangle^{-}\right) \approx \bar{\theta}_{e}^{*},
$$

relating the mass-weighted ${\overline{\theta_{e}}}^{*}=\left\langle\rho \theta_{e}\right\rangle\langle\langle\rho\rangle$ profile along dry isentropes to the average between the poleward and equatorward $\theta_{e}$ profiles, holds. The left- and right-hand sides of this relation are plotted in the left and right panels of Fig. 8. There is clearly good agreement between the two panels of Fig. 8 in midlatitudes, confirming that the directional components yield an accurate decomposition of the mass-weighted climatology in that region. Since the equatorward $\theta_{e}$ profiles are essentially dry in the free troposphere, it suggests that the poleward $\theta_{e}$ profiles alone are sufficient to explain the midlatitudes moist stability.

The poleward $\theta_{e}$ profiles yield moist isentropes that are much less stable than the mass-weighted mean and it is thus expected that the poleward mass fluxes occur at $\theta_{e}$ values that correspond to large deviations from the mean. The deviations can be quantified by comparing the difference $\left|\left\langle\theta_{e}\right\rangle^{+}-\left\langle\theta_{e}\right\rangle^{-}\right|$and the mass-weighted variance of $\theta_{e}$ on dry isentropes; for example,

$$
{\overline{\theta_{e}^{\prime 2}}}^{*}=\left\langle\rho\left(\theta_{e}-{\overline{\theta_{e}}}^{*}\right)^{2}\right\rangle /\langle\rho\rangle \text {. }
$$

Figure 9 shows the ratio of these quantities. In the midlatitudes (except for NH JJA) the difference in directional $\theta_{e}$ profiles is within 2-3 times the standard deviation. In the winter hemispheres a smaller spread exists with the ratio lying between 2.2 and 2.4. Overall, the difference between the two directional $\theta_{e}$ profiles is about 2.3 times the standard deviation of $\theta_{e}$, indicating that the midlatitudes dynamics is dominated by large variance flows.

In their approximation of the circulation on moist isentropes, Pauluis et al. (2011) assumed that the mass flux distribution of $\theta_{e}$ on isobars was Gaussian and according to this assumption the difference between the poleward and equatorward $\theta_{e}$ is proportional to 2.5 times the standard deviation of $\theta_{e}$ [see their Eq. (28)]. The results presented in Fig. 9 qualitatively support this assumption almost everywhere except during NH JJA. Both the present study and Pauluis et al. (2011) show that the moist circulation involves large deviations of $\theta_{e}$ (i.e., large variance) and therefore the circulation cannot be captured using techniques such as the transformed
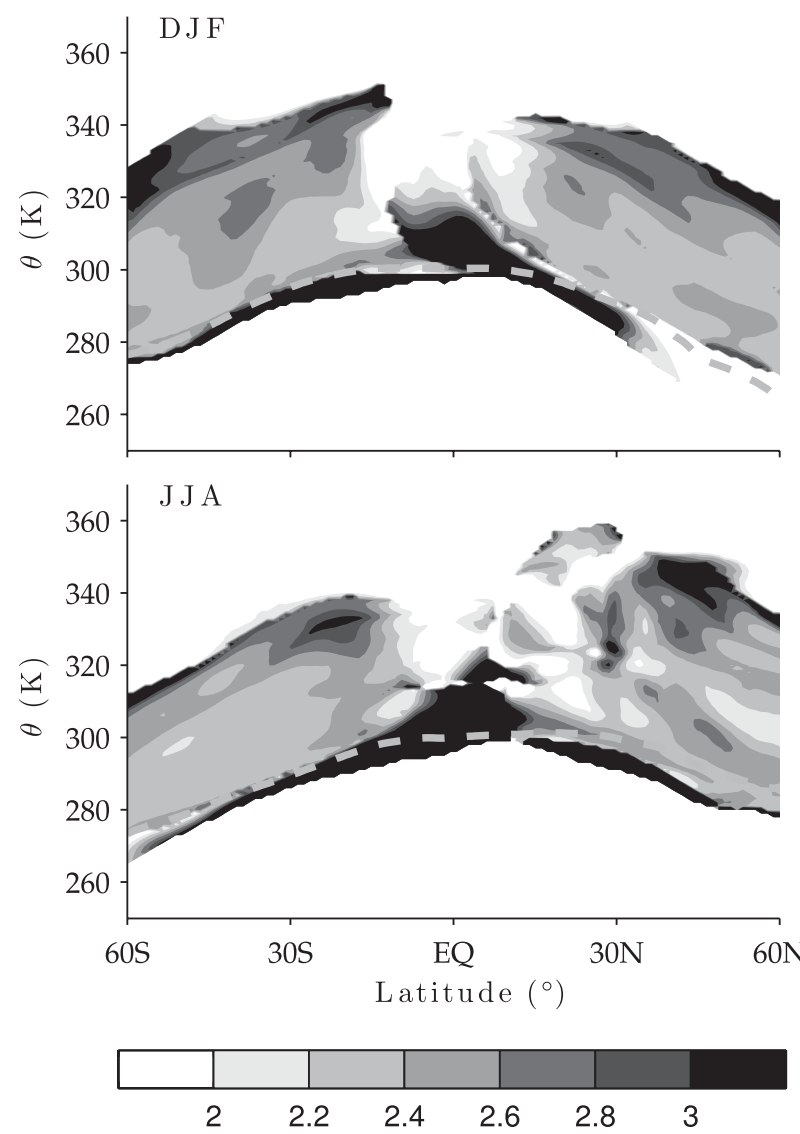

FIG. 9. The ratio of the difference between the poleward and equatorward mean equivalent potential temperature $\mid\left\langle\theta_{e}\right\rangle^{+}-$ $\left\langle\theta_{e}\right\rangle^{-} \mid$and the mass-weighted standard deviation of the equivalent potential temperature on dry potential temperature surfaces ${\overline{\theta_{e}^{\prime 2}}}^{*}$ during (top) DJF and (bottom) JJA.

Eulerian mean, which only uses the mean value of $\theta$ (Andrews et al. 1987).

\section{Reconstructing the moist isentropic circulation}

The results in the previous sections show that the mean meridional mass fluxes on a surface of constant dry potential temperature can be separated into poleward and equatorward directional mass fluxes, which are distinguished in terms of their respective equivalent potential temperatures. It will now be shown that this decomposition also makes it possible to recover the corresponding circulation on moist isentropes.

The moist isentropic circulation measures the net meridional mass fluxes with equivalent potential temperature $\theta_{e}$ less than a fixed value $\theta_{e}^{\prime}$. It can be calculated using the mass flux joint distribution in a procedure similar to the one used to compute the dry isentropic circulation in Eq. (11): 


$$
\begin{aligned}
\Psi_{\theta_{e}}\left(\phi, \theta_{e}^{\prime}\right) & =2 \pi a \cos \phi\left[\int_{0}^{p_{\text {surf }}} v H\left(\theta_{e}^{\prime}-\theta_{e}\right) \frac{d p}{g}\right] \\
& =\int_{0}^{\theta_{e}^{\prime}} \int_{0}^{\infty} M\left(\phi, \theta, \theta_{e}\right) d \theta d \theta_{e} .
\end{aligned}
$$

We drop the primes for the phase space $\theta_{e}$ for the remainder of this paper.

The directional fluxes and directional $\theta_{e}$ can be used to approximate the circulation on moist isentropes by transforming the curves of constant directional $\theta_{e}$ in Figs. 5 and 6 into a vertical axis. To do so, one has to make sure that the associated directional mass fluxes are carried along in such a way that the total $\theta_{e}$ flux is preserved at each latitude. For subtropical poleward fluxes, the directional $\theta_{e}$ does not increase monotonically with $\theta$, leading to the interpretation that poleward motions along moist isentropes are discontinuous. The procedure to approximate the moist isentropic circulation is to effectively "fold" the corresponding fluxes (i.e., summing together low-level air with upper tropospheric air). The poleward branch of the folded moist circulation is thus composed of air masses that have already ascended and of air masses in moist lower troposphere that will ascend if they continue along their poleward path. Similarly, the equatorward fluxes involve the folding of surface and midtropospheric fluxes. This folding leads to cancellations between equatorward and poleward fluxes when the surface equatorward $\theta_{e}$ is comparable to midtropospheric poleward $\theta_{e}$. As it can be seen in bottom panels of Figs. 5 and 6 this effect is important only for latitudes equatorward of $40^{\circ}$.

The procedure to compute the folded streamfunction mimics the computation of the moist streamfunction (11) but instead uses the directional fluxes and their directional $\theta_{e}$ :

$$
\Psi_{\theta_{e}}^{\text {fold }}\left(\phi, \theta_{e}\right)=\int_{0}^{\infty}\langle\rho v\rangle^{+} H\left(\theta_{e}-\left\langle\theta_{e}\right\rangle^{+}\right)-\langle\rho v\rangle^{-} H\left(\theta_{e}-\left\langle\theta_{e}\right\rangle^{-}\right) d \theta
$$

If the decomposition into directional fluxes was exact then $\Psi_{\theta_{e}}^{\text {fold }}$ would be equal to the moist streamfunction (11). In fact, the folded streamfunction (12) approximates the exact streamfunction by reducing the distributions ${ }^{5} M^{+} /\langle\rho v\rangle^{+}$and $M^{-} /\langle\rho v\rangle^{-}$of directional $\theta_{e}$ to $\delta$ functions centered at their respective mean value.

The accuracy of such an approximation can be assessed by comparing it to the exact moist isentropic circulation. Figure 10 shows the exact moist circulation (11) in the left panels and the folded moist circulation (12) in the right panels for both seasons. Qualitatively, the folded circulation is very similar to the exact circulation. The folded circulation is narrower in the $\theta_{e}$ direction, primarily in the subtropics. This is a consequence of cancellations arising from the folding procedure described previously. The folded circulation tends to have sharper gradients, which is a consequence of assuming that directional fluxes occur at their directional $\theta_{e}$ and therefore neglects higher-order moments of the directional distribution. These sharp gradients occur where the directional $\theta_{e}$ is not a monotonic function of $\theta$. This occurs primarily around $30^{\circ}$.

\footnotetext{
${ }^{5}$ On a fixed dry isentrope at a fixed latitude, $M^{+} /\langle\rho v\rangle^{+}$is a function of $\theta_{e}$ that integrates to unity: $\int_{0}^{\infty} M^{+} /\langle\rho v\rangle^{+} d \theta_{e}=1$. If one is given a northward-moving parcel, $M^{+} /\langle\rho v\rangle^{+}\left(\theta_{e}\right) \Delta \theta_{e}$ will be equal to the probability of this parcel having an equivalent potential temperature between $\theta_{e}$ and $\theta_{e}+\Delta \theta_{e}$. A similar explanation stands for $M^{+} /\langle\rho v\rangle^{-}$.
}

To quantify the difference between folded and exact circulations, we calculate the total mass transport (Pauluis et al. 2010):

$$
\Delta \Psi_{\theta_{e}}^{\text {exact }}(\phi)=\max _{\theta_{e}^{\prime}}\left[\Psi_{\theta_{e}}^{\text {exact }}\left(\phi, \theta_{e}^{\prime}\right)\right]-\min _{\theta_{e}^{\prime}}\left[\Psi_{\theta_{e}}^{\text {exact }}\left(\phi, \theta_{e}^{\prime}\right)\right],
$$

and similarly for the folded circulation $\Psi_{\theta_{e}}^{\text {fold }}$. The total mass transport is plotted in Fig. 11, with the solid curve corresponding to the approximate transport and the dashed curve to the exact transport.

For each season, the exact and folded total mass transports are qualitatively similar. The winter season circulation exhibits two maxima: one within the subtropics corresponding to the Hadley cell and one in midlatitudes related to moist eddies. The summer season circulation exhibits only one maximum around $30^{\circ}$. In the $\mathrm{NH}$, this maximum is weak and the total mass transports do not change much with latitude. Overall, the approximate moist isentropic circulation tends to overestimate the true circulation with errors on the order of $10 \%-15 \%$ in the midlatitudes. This overestimation is a direct consequence of the simplification of the directional $\theta_{e}$ distributions into a $\delta$ function, which neglects the variance to leading order.

The good agreement between the moist isentropic circulation and its approximation based on the directional fluxes confirms that the difference in mass transport between the dry and moist isentropic circulations is 

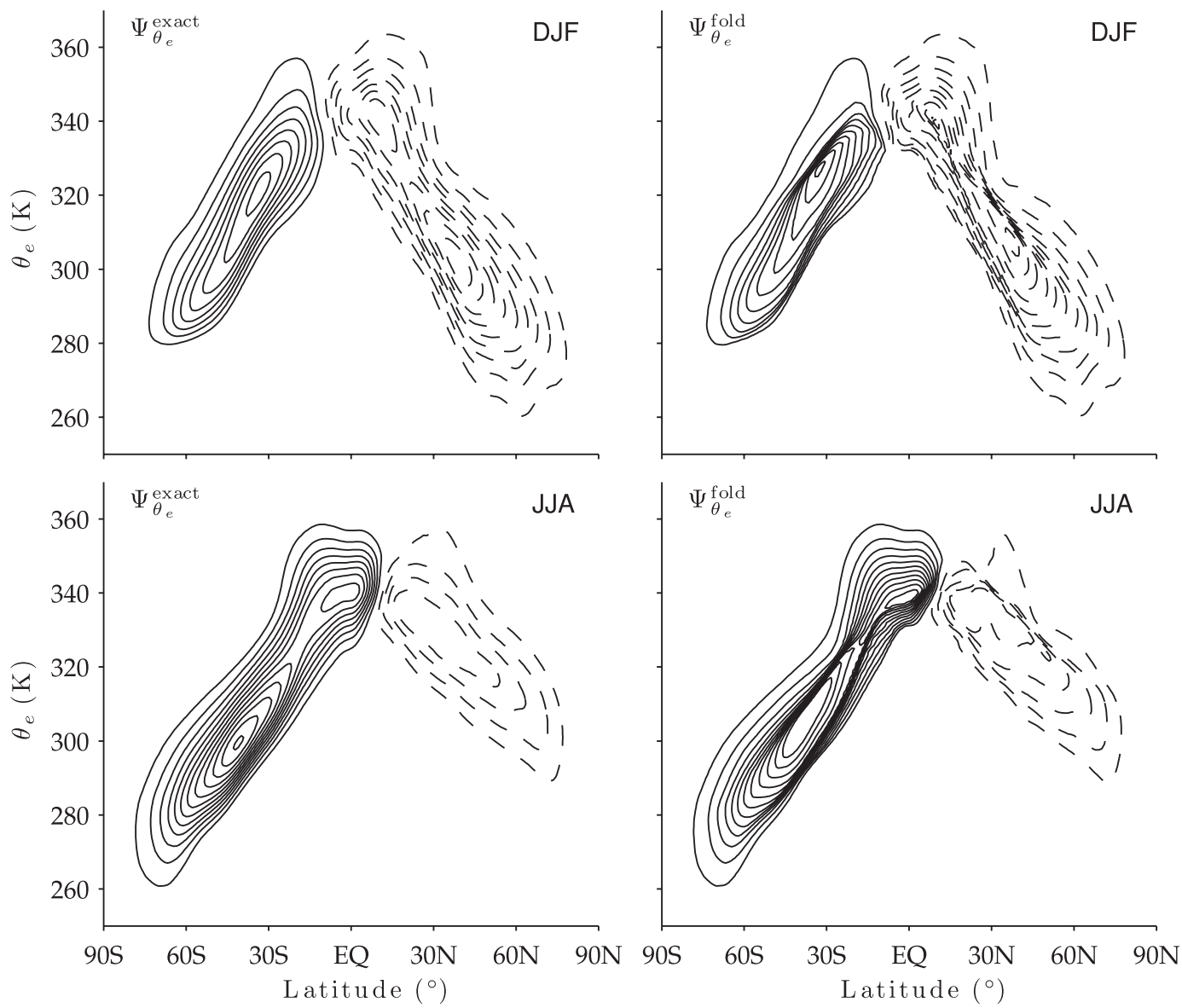

FIG. 10. (left) Moist isentropic circulation due to the exact calculation and (right) the circulation recovered using the directional decomposition during (top) DJF and (bottom) JJA. Contours are as in Fig. 1, from 20 to $240 \mathrm{~Sv}$ in 20-Sv increments.

directly related to the moist recirculation, and thus to the eddy transport of water vapor.

\section{Summary and discussion}

We have presented a new perspective on the global atmospheric circulation that explains the difference in mass transport between the meridional circulations averaged on dry and moist isentropes, a difference that had been initially observed by Pauluis et al. (2008). We introduced a new diagnostic that separates meridional mass fluxes on surfaces of constant dry potential temperature into northward and southward components. The decomposition uses the mass flux joint distribution to identify thermodynamically distinct mass fluxes moving in opposite directions that are responsible for the net poleward transport of water vapor observed in the midlatitudes. We applied the decomposition to reanalysis data and we have shown that the poleward eddy transport of water vapor is achieved through a poleward displacement of moist air parcels balanced by an equatorward displacement of dry air parcels. When these opposing motions occur on the same dry isentrope, this generates a poleward latent heat transport without an

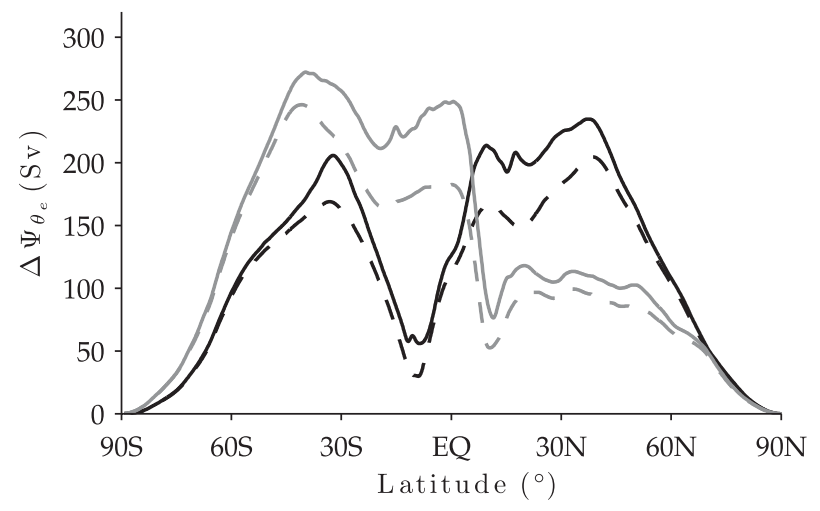

FIG. 11. The total mass transport on moist isentropes in the exact calculation (dashed) and inferred directional calculation (solid). Black curves are for DJF and gray curves for JJA. 
associated sensible heat transport. Since motions must result in a sensible heat transport to be accounted for in the dry isentropic circulation, we conclude that these recirculating motions correspond to a purely moist cycle. We thus call the associated mass transport the moist recirculation [see Eq. (6)].

The opposing mass fluxes forming the moist recirculation occur from the deep subtropics to midlatitudes and across a broad range of dry potential temperatures encompassing surface and upper tropospheric values. The moist recirculation peaks on the equatorward side of the storm tracks where the atmospheric heat transport transitions from being dominated by latent heat transport to being dominated by sensible heat transport. This transition also corresponds to regions of enhanced precipitation associated with the upward ascent of warm, moist subtropical air within the storm tracks, either through deep or slantwise convection.

On the equatorward side of storm tracks, the equivalent potential temperatures in poleward flows indicate that low-level air parcels rise rapidly into the upper troposphere, indicative of fast deep convection. On the poleward side of storm tracks, the same analysis indicates that moist air parcels likely move along slanted moist isentropes. In contrast, the equatorward flows exhibit a marked midtropospheric minimum in $\theta_{e}$, which implies that, as air parcels are brought back toward the equator, they first lose energy through radiative cooling in the free troposphere before being moistened through mixing with moist boundary layer air.

Our results suggest that the vertical $\theta_{e}$ profiles in the poleward flows control the moist stratification in midlatitudes. Indeed, while one can investigate the stability of the atmosphere using the climatological $\theta_{e}$, it would not correspond to the moist stability as experienced by any flows. Instead, we have shown that the climatological $\theta_{e}$ can be seen as an average between the mean $\theta_{e}$ values of the two directional components. The poleward flows experience moist instabilities and the equatorward flows are essentially stable to moist ascents. This implies that the moist stability in midlatitudes is determined by the poleward branch of the flow. Because the poleward $\theta_{e}$ profiles are associated with large moisture variance, it confirms that the directional decomposition effectively captures the large variability intrinsic to midlatitude eddies.

The directional decomposition can be used to obtain an approximate circulation on moist isentropes by assuming that directional components occur at their mean equivalent potential temperature. The approximate moist isentropic circulation is in good qualitative agreement with its exact counterpart but it is quantitatively biased because directional $\theta_{e}$ profiles neglect higher $\theta_{e}$ moments in directional distributions. The approximate circulation has sharper gradients than the circulation on moist isentropes. These sharper gradients are associated with a vanishing moist stratification in directional $\theta_{e}$ profiles. This confirms the interpretation that the additional mass transport in the circulation on moist isentropes is associated with net poleward mass fluxes of warm moist air near the surface.

Another approximate circulation on moist isentropes has been simultaneously derived by Pauluis et al. (2011) where it is shown that in order to accurately compute the moist circulation one must take into account large zonal variances in equivalent potential temperature, which are found throughout the troposphere. In the analysis presented here, the large differences between directional $\theta_{e}$ profiles reinforce the importance of the variance. While the approximate circulation in Pauluis et al. (2011) underestimates the total mass transport, the approximate folded circulation presented here provides an upper bound estimate.

The decomposition in this paper highlights the need to better understand what sets the poleward $\theta_{e}$ profiles. While the directional $\theta_{e}$ profiles have been connected to surface conditions and related to the variance of moisture on dry potential temperature surfaces, we do not have presented a theory explaining why this should be true. In a companion paper, we develop a theoretical model that helps describe the poleward $\theta_{e}$ profiles in terms of boundary layer moisture and temperature variability. This theory is then used to explain what sets the relative strength of the moist isentropic circulation to the dry isentropic circulation, thus offering a new interpretation of the impacts of climate change on the midlatitude storm tracks. Laliberté and Pauluis (2010) show that in the Intergovernmental Panel on Climate Change Fourth Assessment Report (IPCC AR4) simulations, the winter mass transport on dry isentropes in midlatitudes weakens faster than the corresponding mass transport on moist isentropes. These results imply an enhancement of the winter moist recirculation (i.e., an increase in the mass of air being recirculated by moist eddies across the storm tracks). Understanding the relationship between moist recirculation and eddy variability is a key issue for our broader understanding of midlatitudes storm tracks and for our ability to predict the impacts of future climate change on the hydrological cycle in the midlatitudes.

Acknowledgments. We thank the Computational and Information Systems Laboratory at the National Center for Atmospheric Research for providing the ECMWF data. Frédéric Laliberté was supported by a McCracken Fellowship from the New York University and by the 
NSERC/CRSNG doctoral fellowship program. Tiffany Shaw acknowledges support from the NSERC/CRSNG through a postdoctoral fellowship. This work was supported by the NSF under Grant AGS-0944058.

\section{REFERENCES}

Andrews, D., C. Leovy, and J. Holton, 1987: Middle Atmosphere Dynamics. International Geophysical Series, Vol. 40, Academic Press, 489 pp.

Czaja, A., and J. Marshall, 2006: The partitioning of poleward heat transport between the atmosphere and ocean. J. Atmos. Sci., 63, $1498-1511$

Emanuel, K., 1994: Atmospheric Convection. Oxford University Press, $580 \mathrm{pp}$.

Gallimore, R. G., and D. R. Johnson, 1981: A numerical diagnostic model of the zonally averaged circulation in isentropic coordinates. J. Atmos. Sci., 38, 1870-1890.

Held, I., and T. Schneider, 1999: The surface branch of the zonally averaged mass transport circulation in the troposphere. J. Atmos. Sci., 56, 1688-1697.

Johnson, D., 1989: The forcing and maintenance of global monsoonal circulations: An isentropic analysis. Advances in Geophysics, Vol. 31, Academic Press, 43-316.

Karoly, D. J., P. C. McIntosh, P. Berrisford, T. J. McDougall, and A. C. Hirst, 1997: Similarities of the Deacon cell in the Southern Ocean and Ferrel cells in the atmosphere. Quart. J. Roy. Meteor. Soc., 123, 519-526.
Laliberté, F., and O. Pauluis, 2010: Winter intensification of the moist branch of the circulation in simulations of 21st century climate. Geophys. Res. Lett., 37, L20707, doi:10.1029/ 2010GL045007.

Lorenz, E., 1967: The nature and the theory of the general circulation of the atmosphere. WMO Rep. 218, $161 \mathrm{pp}$.

Pauluis, O., A. Czaja, and R. Korty, 2008: The global atmospheric circulation on moist isentropes. Science, 321, 1075-1078, doi:10.1126/science.1159649.

$[,-$, and _ 2010: The global atmospheric circulation in moist isentropic coordinates. J. Climate, 23, 3077-3093.

— T. Thaw, and F. Laliberté, 2011: A statistical generalization of the transformed Eulerian-mean circulation for an arbitrary vertical coordinate system. J. Atmos. Sci., 68, 1766-1783.

Pierrehumbert, R. T., 1998: Lateral mixing as a source of subtropical water vapor. Geophys. Res. Lett., 25, 151-154.

Roma, A., C. Peskin, and M. Berger, 1999: An adaptive version of the immersed boundary method. J. Comput. Phys., 153, 509-534.

Schneider, T., 2006: The general circulation of the atmosphere. Annu. Rev. Earth Planet. Sci., 34, 655-688.

_, K. Smith, P. O'Gorman, and C. Walker, 2006: A climatology of tropospheric zonal-mean water vapor fields and fluxes in isentropic coordinates. J. Climate, 19, 5918-5933.

Trenberth, K. E., and D. P. Stepaniak, 2003: Covariability of components of poleward atmospheric energy transports on seasonal and interannual timescales. J. Climate, 16, 3691-3705.

Uppala, S. M., and Coauthors, 2005: The ERA-40 Re-Analysis. Quart. J. Roy. Meteor. Soc., 131, 2961-3012.

Yang, H., and R. Pierrehumbert, 1994: Production of dry air by isentropic mixing. J. Atmos. Sci., 51, 3437-3454. 